\title{
Supplementary Discussion and Figures
}

\section{Change of local density along the QD}

For further comparison of experimental and theoretical data, we provide in Supplementary Figure 1 the separate linear profiles of calculated states $\alpha, \beta, \gamma$, and $\delta$. The 1:1 mixing of pairs $\alpha$ and $\beta, \gamma$ and $\delta$, respectively, is shown on the right hand side of Fig. 3c. The experimental linear profiles appearing on the left hand side of Fig. 3c were obtained by averaging the differential conductance in the transverse direction in the spatial regions shown in Supplementary Figure 2.

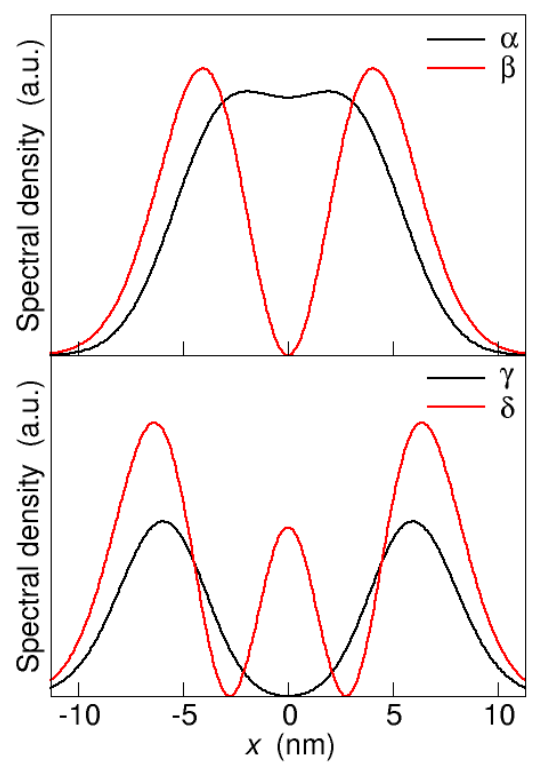

Supplementary Figure 1. Separate profiles extracted from $\alpha$ and $\beta$ (top) and $\gamma$ and $\delta$ (bottom) predicted maps of Fig. 3b. 

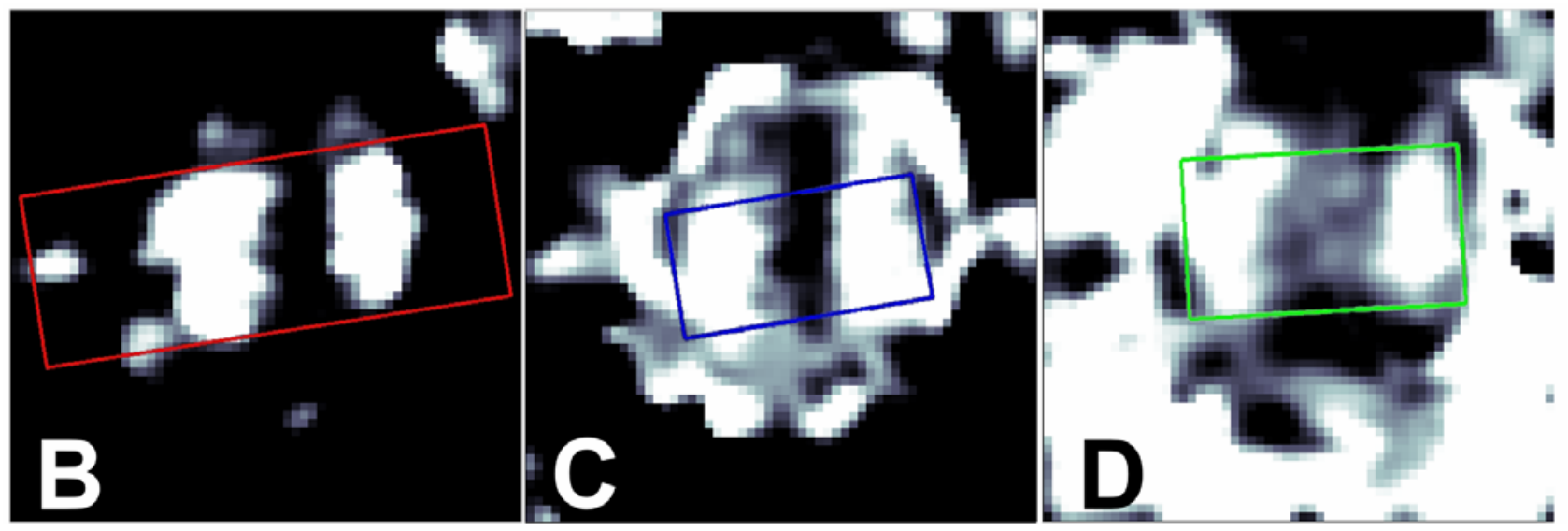

Supplementary Figure 2. STS spatial maps (same set of data as the maps of Fig. 2) of a single representative dot recorded at the voltages 1040 (B), 1140 (C), and 1370 (D) $\mathrm{mV}$, respectively. The size of all maps is $30 \times 30 \mathrm{~nm}$. $I_{\text {stab }}=100 \mathrm{pA}, V_{\text {stab }}=1500 \mathrm{mV}, V_{\text {mod }}=4 \mathrm{mV}$. White (black) colour stands for high (low) values of $(d I / d V) /(I / V)$. The colored rectangles show the spatial regions where the profiles in Fig. 3c were averaged.

\section{Interpretation of state $C$}

We associate states $\mathrm{C}$ and $\mathrm{D}$ to the tunneling process $N=1 \rightarrow N=2$. In particular, since the peak FWHM in the WF mapping is around $50 \mathrm{mV}$, the energy resolution is not sufficient to distinguish the calculated ground- $\rightarrow$ (ground-) excited-state transitions (right column of Fig. 3b) $\alpha$ from $\beta$ (experimental state C) and $\gamma$ from $\delta$ (D). By assuming a value 2.6 for the voltage/energy conversion factor, the $\mathrm{B}$ to $\mathrm{C}$ energy splitting is about $38 \mathrm{meV}$, quite close to that of optical phonons in the InAs/GaAs system. However, we exclude that state $\mathrm{C}$ is a phonon replica of B (involving the emission of an optical phonon of the QD or of the GaAs layer below) for the following three reasons.

(i) The dependence of the STS spectra on the stabilization current suggests that state C must be associated to the tunneling process $N=1 \rightarrow N=2$ since it only appears at higher $I_{\text {stab }}$ where state B then disappears (the STS levels measured at increasing values of the current correspond to higher values of QD occupancy). On the other hand, we would not expect state B to disappear if it were the main peak associated to elastic tunneling.

(ii) A phonon replica would presumably appear as a small side peak due to a reduction of transition probability (it is a second order process), while B and C states exhibit comparable spectral densities (see Fig. 2).

(iii) We did not observe any phonon replica of state $s$. 
Moreover, the small energy splitting between B and C states along with the small blue shift of the whole spectra to higher energies on the QD sides accounts for the high intensity regions on the QD sides visible in the STS spatial map of state C (Figure 2b and Supplementary Figure 2), which belong to state B.

\section{Effect of the dielectric mismatch among quantum dot, vacuum, and STS tip}

As mentioned in the main text, the dielectric mismatch between QD and vacuum is responsible for the appearance of negative image charges on the dot surface, which increases the effective Coulomb repulsion among electrons in the QD. This effect is described in a simple way by the effective value $\kappa=4$ of the relative dielectric constant used in the FCI calculation: the smaller $\kappa$, the larger the Coulomb matrix elements, the stronger the electron correlation. A concern regards the presence of the STS tip, which in principle could screen the interaction and wipe off the effect. In this section we discuss the competing effects of the QD-vacuum and vacuum-electrode interfaces, and conclude that the Coulomb repulsion is significantly enhanced even in the presence of the STS tip.

The estimate of the effective Coulomb repulsion relies on the calculation of the Green's function as defined in classical electrostatics [see e.g. Jackson, J. D. Classical Electrodynamics, Chapters 2 and 3 (Wiley, New York, 1975), and Refs. 26, 27]. Since only the simplest geometries allow for an almost analytical solution of the problem, here we consider a highly schematic model of the dielectric environment consisting in three parallel layers indefinitely extended in the lateral directions, namely two dielectrics (with relative dielectric constants $\kappa_{1}$ and $\kappa_{2}$, respectively) and a metal. Let $z$ be the axis perpendicular to the perfectly flat interfaces, and locate the dielectric 1 / dielectric 2 interface at $z=0$ and the dielectric $2 /$ metal interface at $z=L$ [the limit $z \rightarrow-\infty \quad(+\infty)$ corresponds to the bulk dielectric 1 (metal)]. The calculation proceeds through the lines indicated e.g. in Panofsky, W. K. H. \& Phillips, M. Classical Electricity and Magnetism, Sec. 5.8 (Addison-Wesley, New York, 1962), based on the matching of the solutions of the Laplace equation in the three media by exploiting the lateral translational symmetry. Here we only state the final result for the effective Coulomb repulsion $V_{\text {eff }}(\rho, z)$ between two electrons, located at relative lateral distance $\rho$ and both placed at $z<0$ :

$$
\begin{gathered}
V_{\mathrm{eff}}(\rho, z)=\frac{e^{2}}{\kappa_{1}} \int_{0}^{\infty} d k\left[1+f_{z}(k)\right] J_{0}(k \rho), \\
f_{z}(k)=\frac{\left(\kappa_{2}-\kappa_{1}\right) \exp [2 k(z+L)]+\left(\kappa_{2}+\kappa_{1}\right) \exp (2 k z)}{\kappa_{1}[1-\exp (2 k L)]-\kappa_{2}[1+\exp (2 k L)]},
\end{gathered}
$$

where $J_{0}(x)$ is the zero-order Bessel function of the first kind. Note the two important cases: (i) With $\kappa_{1}=\kappa_{2}, L \rightarrow \infty$, we have $f_{z}(k) \rightarrow 0$ and recover the bulk dielectric result $V_{\text {eff }}(\rho, z) \rightarrow e^{2} /\left(\kappa_{1} \rho\right)$. (ii) 
With $L \rightarrow \infty$ we recover the result for the two-dielectric problem, $V_{\text {eff }}(\rho, z) \rightarrow e^{2} /\left(\kappa_{1} \rho\right)+e^{2}\left(\kappa_{1}-\kappa_{2}\right) /\left[\kappa_{1}\left(\kappa_{1}+\kappa_{2}\right)\left(\rho^{2}+4 z^{2}\right)^{1 / 2}\right]$, given by the sum of the bulk pair interaction plus the repulsion with the image charge placed at vertical distance $|2 z|$. The maximum value that $V_{\text {eff }}$ may reach is obtained in the latter case by putting $\kappa_{1} / \kappa_{2} \rightarrow \infty$ and $z=0$, resulting in twice the bulk value (such an upper bound is specific to the planar plate geometry).

To proceed further, we assume that electrons are laterally confined by a circular harmonic potential $V_{\text {conf }}(\rho)=m \omega^{2} \rho^{2} / 2$, where $\hbar \omega$ is the confinement energy and $m$ is the effective electron mass. The (Fock-Darwin) ground state wave function of the single-particle problem, neglecting the motion along $z$, is the $s$-like Gaussian $\varphi_{s}(\rho)=\exp \left(-\rho^{2} / 2 \ell_{\mathrm{QD}}^{2}\right) / \sqrt{\ell_{\mathrm{QD}}^{2} \pi}$, where $\ell_{\mathrm{QD}}=\sqrt{\hbar / m \omega}$ is the characteristic lateral extension of the orbital. In order to quantify the strength of electron correlation, we compute the Hubbard-like electrostatic repulsion $U$ referred to the $s$-orbital:

$$
U=\iint d \vec{\rho}^{\prime} d \vec{\rho}\left|\varphi_{s}\left(\rho^{\prime}\right)\right|^{2} V_{\text {eff }}\left(\left|\vec{\rho}^{\prime}-\vec{\rho}\right|, z\right)\left|\varphi_{s}(\rho)\right|^{2} .
$$

After separation of the relative and center-of-mass coordinates, we eventually obtain an expression convenient to numerical evaluation:

$$
U=\frac{e^{2}}{\kappa_{1} \ell_{\mathrm{QD}}} \int_{0}^{\infty} d x\left[1+f_{z}\left(x / \ell_{\mathrm{QD}}\right)\right] \exp \left(-x^{2} / 2\right) .
$$

To apply the latter equation to the actual experimental geometry, we introduce effective thicknesses for the QD and vacuum regions, respectively. We proceed as follows: We model the QD as half an ellipsoid with basal semi-axes $a=b=15 \mathrm{~nm}$, vertical semi-axis $c=5 \mathrm{~nm}$, and define the equivalent QD height $h_{\text {equiv }}$ as the height of the cylinder of radius $a$ and same volume as that of the ellipsoid: $h_{\text {equiv }}=2 c / 3$. We then assume that electron motion is confined in the middle of the QD, $z=-h_{\text {equiv }} / 2$, and put $L=1$ $\mathrm{nm}$. The value of $U$ obtained from the above parameters has to be compared with the reference case in which the STS tip is absent, i.e. $L \rightarrow \infty$. The relative reduction turns out to be $29 \%$ (27\%) for GaAs (InAs). Nevertheless, $U$ is still 5\% (9\%) larger than the value corresponding to a unique bulk, i.e. $\kappa_{1}=\kappa_{2}, L \rightarrow \infty$. This demonstrates that the enhancement of electron correlation due to the dielectric mismatch survives to the screening effect of the STS tip. Besides, correlation effects are expected to be much larger than those estimated within this simple model. In fact, while in the planar plate geometry the maximum value of $U$ attainable is only twice the bulk value, in the case of a spherical dot in the vacuum $U$ may be several times larger than the bulk value without any interface [Franceschetti, A., Williamson, A. \& Zunger, A. Addition spectra of quantum dots: the role of dielectric mismatch. J. Phys. Chem. B 104, 3398-3401 (2000)]. The representation of the actual experimental setup should fall 
somewhere in the middle between the limiting cases of the planar plate and spherical geometries, respectively. In addition, in our calculation the effective dot-tip distance is likely underestimated.

In conclusion, on the basis of a rough estimate we believe the actual value of $\kappa$ employed in the FCI calculation is sensible. 